\title{
Block structured dynamics and neuronal coding
}

\author{
J. M. González-Miranda \\ Departamento de Física Fundamental, Universidad de Barcelona, Avenida Diagonal 647, 08028 Barcelona, Spain
}

(Received 10 May 2005; revised manuscript received 16 September 2005; published 18 November 2005)

\begin{abstract}
When certain control parameters of nervous cell models are varied, complex bifurcation structures develop in which the dynamical behaviors available appear classified in blocks, according to criteria of dynamical likelihood. This block structured dynamics may be a clue to understand how activated neurons encode information by firing spike trains of their action potentials.
\end{abstract}

DOI: 10.1103/PhysRevE.72.051922 PACS number(s): 87.19.La, 05.45.-a, 87.10.+e, 87.17.Nn

\section{INTRODUCTION}

The neuronal code problem [1-7] is a major issue in today's neuroscience. A common assumption in the scientific community is that, in the nervous system, information is transmitted along the neurons axon by means of trains of spikes of the action potential. The problem that then emerges is how the information carried by the neuron is encoded in these trains. Because action potential firing is quite irregular, a first classical hypothesis is that the code is simply given by the firing rate averaged in an appropriate time interval. Recently, based on experiments and computer simulations, a more sophisticated theory is emerging which asserts that the information is coded in the temporal structures of the firing sequence [3-5], and that synchronization between different neurons plays a relevant role $[1,2]$. These new hypotheses require that accurate and reproducible firing structures can be produced in the nervous system [6,7]. Unveiling the mechanisms that allow the generation of properly structured trains of spikes is then a problem of major importance.

By means of a study of the Hindmarsh-Rose neuron model [8], we will show that there is a particular type of dynamic behavior, which we call block structured dynamics, that provides the background for a potential neural coding mechanism. The important feature of these structures is that the system bifurcation diagram can be divided into disjoint blocks, each corresponding to a family of dynamical behaviors sufficiently alike between them as to be considered as the same dynamics in a coarse description and different enough from the dynamics of other blocks as to be unambiguously distinguished from one another. This feature allows us to imagine reliable coding mechanisms whose foundations lie in three important general properties of life: variability (no two individuals of a given population are exactly alike), noise (life occurs in a noisy environment), and self-regulation (living beings are able to maintain vital parameters within controlled limited ranges of values).

The Hindmarsh-Rose model [8] results from a simplification of the current-voltage relationships of the HodgkinHuxley model of neuronal activity [9]. It provides a realistic description of the firing dynamics of neurons observed in molluscans, crustaceans, and vertebrates $[8,10]$. Therefore, this model has been used to study different aspects of neuronal dynamics such as transitions between different firing regimes [11], the effect of noise on neuronal signal transduction [12] and on synchronization [13], the synchronization dynamics of two coupled neurons $[14,15]$, the collective dynamics of populations of coupled neurons $[16,17]$, and the information exchange in neuronal communication [18]. Moreover, we have found that it displays very neatly the block structure dynamics, which is the object of this paper. Because of all this, we have chosen it for the research reported here.

The layout of the paper is as follows. Block structured dynamics in the Hindmarsh-Rose model will be described in Sec. II, the effect of external noise will be studied in Sec. III, and ideas on neuronal coding will be discussed in Sec. IV. Finally, results for other models of excitable cells will be discussed in Sec. V, and the paper will end in Sec. VI with some comments and conclusions.

\section{BLOCK STRUCTURED DYNAMICS}

The relevant observable that describes the dynamical state of a single neuron is the membrane potential, $x$. According to the Hindmarsh-Rose model [8], its dynamics is given by the following system of stochastic differential equations written in dimensionless form:

$$
\begin{gathered}
d x=\left(y+3 x^{2}-x^{3}-z+I\right) d t+b d W_{t}, \\
d y=\left(1-5 x^{2}-y\right) d t, \\
d z=\{r[4(x+8 / 5)-z]\} d t,
\end{gathered}
$$

where $t$ is time; $x, y, z$ are dynamical variables that describe the dynamic state of the neuron; and $W_{t}$ a standard Wiener process [19], which stands for an external noisy environment. The membrane potential as a function of time is given by $x(t)$. Charge transport processes across the axon membrane are assumed to occur through fast channels $\left(\mathrm{Na}^{+}\right.$and $\mathrm{K}^{+}$ions) as modeled by $y(t)$ and through slow channels (other ions) which are taken into account through $z(t)$. The external electrical current applied, $I$, and the rate of change of $z(t), r$, are the control parameters of the model used often. The Hindmarsh-Rose model is derived from a mapping [20] of the dynamics of the Hodgking-Huxley model [9] to a dimensionless mathematical model of a nonlinear oscillator. The units of the quantities in Eqs. (1)-(3) can then be estimated from this mapping. Using the results in Refs. [8,20], the estimate for the units of time $\tau$, voltage $\nu$, and density 
current $\iota$, are $\tau \approx 1.0 \mathrm{~ms}, \nu \approx 25 \mathrm{mV}$, and $\iota \approx 2.5 \mathrm{~mA} / \mathrm{cm}^{2}$. Units for other quantities can be inferred from them. We have solved numerically Eqs. (1)-(3) by means of a EulerMaruyama scheme [19] with time step $\Delta t=0.0001$. The noisy environment has been modeled by $b \Delta W_{t}$ $=\sqrt{\Delta t} D \xi(t) \Delta t$, with $\xi(t)$ being a white Gaussian noise with zero mean and variance 1 , and the strength of the noise being measured by the parameter $D$.

The results presented in this paper are for fixed $I=3.28$, and $r$ changing as the control parameter. This parameter measures the efficiency of the ionic transport across the nerve cell membrane through channels different from the fast $\mathrm{Na}^{+}$and $\mathrm{K}^{+}$channels. Thus, it describes the average effect of a variety of channels that may be voltage sensitive as well as chemically activated. Then, the variation of $r$ can have two meanings. For a single cell, this will be given by the fraction of these channels that are simultaneously opened during effective nerve operation. This may change, for example, by changing the concentration of some appropriate channel activating or deactivating chemical. In a population of cells, different values of $r$ would mean to consider different cells of the population, having each cell its own characteristic density of channels, which will define a particular average value for $r$ in each neuron.

The set of parameter values implied in Eqs. (1)-(3) and the value of $I$ chosen are within the range of values proposed by Hindmarsh-Rose [8] and are considered realistic. We change here the value of $r$ within a range that includes but exceeds that studied by Hindmarsh-Rose [8]. However, because of the meaning of $r$ as an average of the ion permeability of several different channels, and the well-known experimental fact that neurons are characterized by a great variability among individuals, we mean that this extension is justified and useful.

Bifurcation diagrams and Lyapunov spectra provide a global view of the dynamical behaviors available to a system when a control parameter is modified. In the present case, we have obtained them numerically by running Eqs. (1)-(3) for $D=0$. The feature of the action potential dynamics that is implied in neural coding is the sequence of spikes, which is described by the succession of interspike intervals (ISI), denoted by $\left\{T_{j=1,2, \ldots, N}\right\}$. We have obtained bifurcation diagrams from the ISI values $T$, and Lyapunov exponents $\lambda$ from standard techniques. The results presented in Fig. 1 show that the bifurcation diagram and Lyapunov spectrum observed are more complex than what is usually seen in three-dimensional flows. The well-known structure of the bifurcation diagram of the logistic map appears as the basic constitutive element of the complexity presented in Fig. 1. The main features of this standard structure, which has been widely studied in the literature [21], can be seen in Figs. 1(a) and 1(b) for $r>0.01025$ : an inverted period doubling route to chaos which starts at $r \approx 0.036$ (out to the right of the plot) and ends at $r \approx 0.01025$.

When $r$ decreases from $r \approx 0.01025$, the bifurcation diagram and the Lyapunov spectrum become more complex. The dependence of the transversal Lyapunov exponent on $r$ results from the repetition, with a change of scale, of the characteristic structure of the logistic map. We describe the complexity observed in the bifurcation diagram by means of

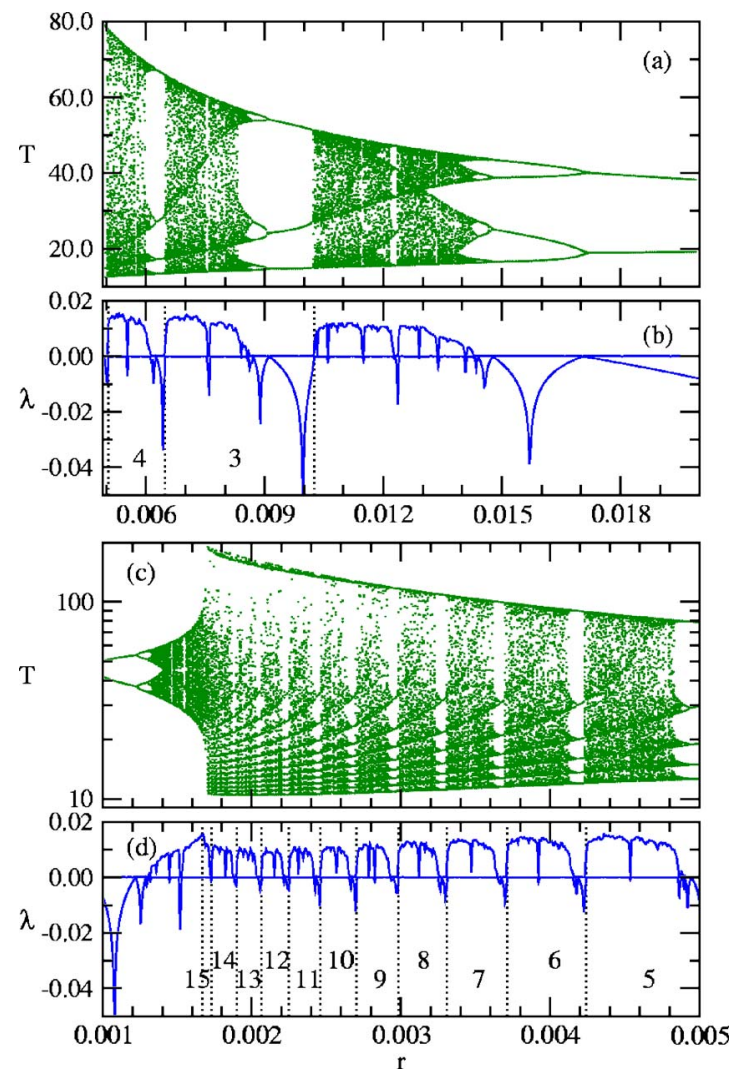

FIG. 1. (Color online) The Bifurcation diagram (a), (c), and the two largest Lyapunov exponents (b), (d) of the Hindmarsh-Rose neuron model for values of $r$ in the intervals: (a), (b) [0.0049, $0.0200]$, and (c), (d) $[0.0010,0.0050]$. Blocks are identified by their numbers $p$ and separated by vertical dotted lines in (b), (d). Quantities plotted are dimensionless. $T$ is measured in units of time $\tau$, and $\lambda$ and $r$ in units of reciprocal time $\tau^{-1}$.

the division of the whole diagram in a sequence of blocks. This is done by dividing the $r$ axis in a series of intervals limited by points where chaos-periodicity transitions occur by means of a saddle-node bifurcation. Each of these intervals is what we call a block and corresponds with a whole repetition of a logistic map structure in the Lyapunov spectrum plot.

These intervals are defined in such a way that the bifurcation diagram that corresponds to each block can be seen as being made up by the superposition of $p$ logistic-map-like structures, with $p$ in each block a unit larger than in the previous block. Being $p$ and $p+1$, the periodicities observed in the nonchaotic left sides of the upper and the lower points of an interval, the correspondent block will be identified by the number $p$ at the upper limit. For example, in Figs. 1(a) and 1(b) a block with $p=3$ begins at $r \approx 0.01025$ and ends at $r \approx 0.00648$, where a new block with $p=4$ starts.

The decrease of the control parameter causes a cascade of period adding bifurcations, as new blocks are developed [Figs. 1(c) and 1(d)]. This cascade is not infinite, but ends at certain maximum $p$, which in the case of the example studied here is $p=15$. However, in similar calculations performed with different values of $I$, we found this number to be different, being larger for smaller values of $I$. When the maximum 

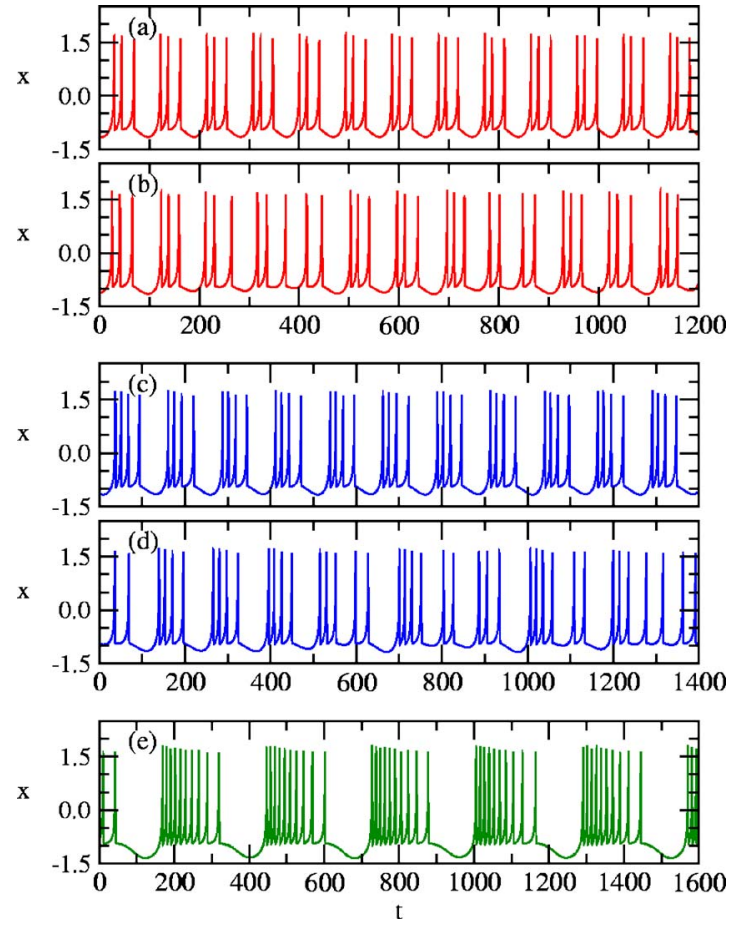

FIG. 2. (Color online) Time series for the membrane potential computed for values of $r$ that lead to dynamics in block $p=3$, (a) $r=0.0095$ and (b) $r=0.0080$; in block $p=4$, (c) $r=0.0062$ and (d) $r=0.0056$; and in block $p=10$, (e) $r=0.00265$. Quantities plotted are dimensionless, $t$ is measured in units of time $\tau$, and $x$ in units of voltage $\nu$.

$p$ is reached, further decrease of $r$ results in a continuous interior crisis, which is a chaos-chaos transition (previously studied in Ref. [11]), which separates the bursting and the spiking dynamics in the Hindmarsh-Rose model.

The bifurcation diagram of a dynamical system provides a global picture of the dynamical states available to that system. In particular, for neurons it tells us which are the firing regimes available. Spike firing is the mechanism that the neurons use to encode information; consequently, bifurcation diagrams of neurons contain fundamental information for neuronal coding. We illustrated this in Fig. 2 by means of plots of $x(t)$ for different values of $r$. The time series shown in Figs. 2(a), 2(c), and 2(e) have been computed for $r$ in the periodic regimes within the blocks $p=3, p=4$, and $p=10$, respectively. They show that the dynamics is made by a sequence of identical bursts of spikes, each burst containing as many spikes as the periodicity $p$ of the block. The time series in Figs. 2(b) and 2(d) are for the chaotic regimes within the blocks $p=3$ and $p=4$. In these cases, $x(t)$ is given by an aperiodic sequence of burst with different numbers of spikes each. However, the block periodicity is conserved in the sense that bursts with a number of spikes equal to the periodicity of the block are dominant. The above bifurcation diagrams are then closely related to neural coding because each block has associated a characteristic structure of the action potential firing, and neuronal information is assumed to be encoded in this kind of structure.

\section{BLOCK STATISTICS}

To show how this block structure can be used for neuronal coding, we rely on the fact that within each block there is a cascade of bifurcations in which, as $r$ decreases, orbits of low periodicity lose stability in favor of orbits of higher periodicity until a chaotic regime is reached where all these periodic orbits become an unstable set [22]. External noise will tend to even out the dynamics within each block [21] by allowing the system to move in the neighborhood of these unstable periodic orbits. Because each block has a different basic periodicity, we expect that, in a noisy environment, there will be observables that, despite the smoothing caused by the noise, will retain memory of the block structured dynamics. Therefore, we consider the full stochastic dynamics $(D \geqslant 0)$ given by Eqs. (1)-(3) and focus on statistical quantities that are commonly used to characterize the sequences of ISI.

We have studied the moments of a statistical distribution: mean, variance, and skewness [23], as well as others like the coefficient of variation which is commonly used [24] in this context. We present now the results for the variance, which is a quantity frequently used in experiments. This is defined as

$$
V=\frac{1}{N-1} \sum_{j=1}^{N}\left(T_{j}-\langle T\rangle\right)^{2}
$$

with $\langle T\rangle=N^{-1} \sum_{j=1}^{N} T_{j}$ being the mean of the distribution. The dependence of $V(r)$ on $D$ appears in Fig. 3.

For the free noise case [upper curve in Fig. 3(a)], the dependence of $V$ on $r$ displays a block structure according to those in Fig. 1. Within each block, the variation of $V(r)$ has roughly the shape of a wave with a minimum, $V_{m}^{(p)}$ $=V\left(r_{m}^{(p)}\right)$, and a maximum, $V_{M}^{(p)}=V\left(r_{M}^{(p)}\right)$, occurring at $r_{m}^{(p)}$ and $r_{M}^{(p)}>r_{m}^{(p)}$, respectively. This structure is associated to the period doubling transition between regular and chaotic dynamics inside the block with the lower values of $V$ corresponding to chaotic behavior. Moreover, the center of oscillation of each wave decreases monotonously with increasing $r$, which results in an overall negative slope in the plot of $V$ versus $\ln (r)$.

The effect of external noise is to smooth this structure until $V$ becomes a monotonous decreasing function of $r$ [curves from top to bottom in Fig. 3(a)]. In this transition, we have a low noise regime where the wave structure, although softened, persists. In this regime, there are still minima and maxima, $V_{m}^{(p)}$ and $V_{M}^{(p)}$, for $V(r)$ within each block, but the values of these two extrema approach each other with little change in their positions. At certain value of the noise strength characteristic of each block, $D_{C}^{(p)}$, the extrema disappear within the block and $V(r)$ becomes monotonously decreasing. The values $D_{C}^{(p)}$ are larger as the blocks come closer to the limits of the $r$ interval, where the block structured dynamics occurs. For $V$, they change smoothly from $D_{C}^{(6)} \approx 0.0015$ to $D_{C}^{(14)} \approx 0.0055$, being the values of $D_{C}^{(p)}$ for consecutive blocks close to each other. So that given a block $p$, there is an interval of noise intensities where, for at least a series of several consecutive blocks, $V(r)$ has roughly the shape of a stairs profile, with plateaus having approximately 


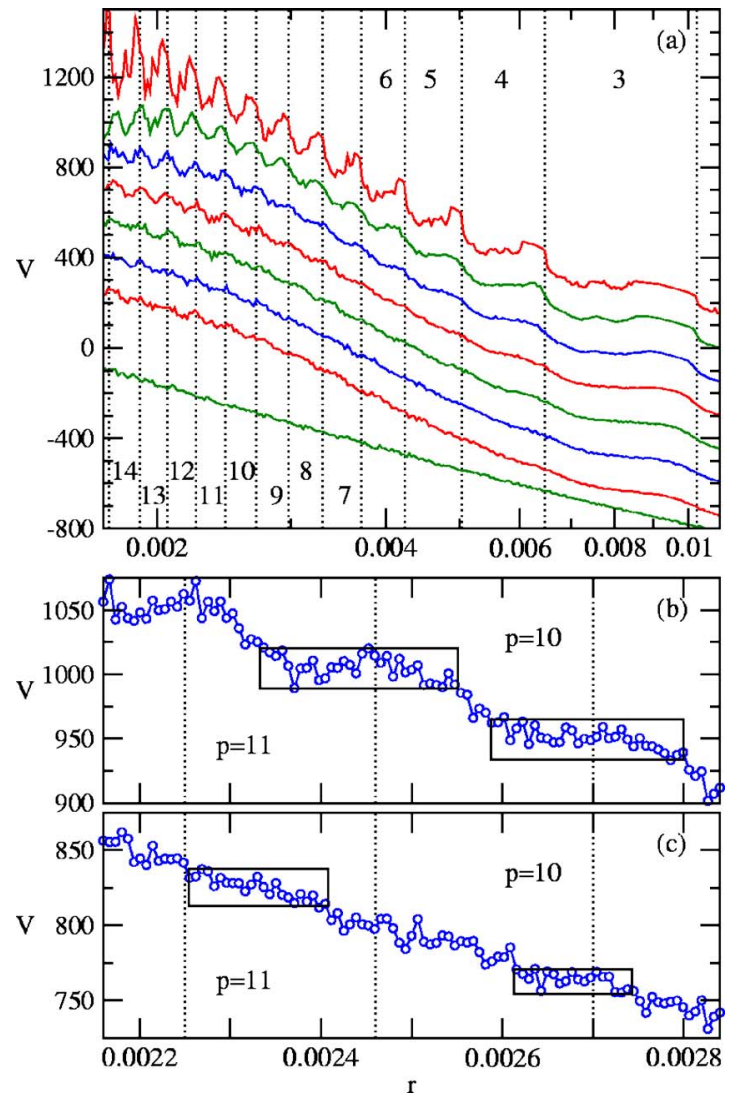

FIG. 3. (Color online) (a) Dependence of $V$ on $r$ for a series of seven values of $D$, evenly spaced an amount 0.001 , starting at $D=0$ and ending at $D=0.006$, plus an additional plot for $D=0.100$. The plots for the different values of $D$ have been shifted down an amount 150.0 from its previous element in the series to separate them and make the figure clear. Plots of $V(r)$ at $p=10$ and $p=11$ for (b) $D=0.0048$ and (c) $D=0.1$. In these last two cases, boxes of width $\Delta^{(p)}$ and height $\delta^{(p)}$ are also plotted. Quantities plotted are dimensionless, $V$ is measured in units of time squared $\tau^{2}$, and $r$ in units of reciprocal time $\tau^{-1}$.

the width of the block, and the fluctuations of $V(r)$ being smaller than the steps between consecutive plateaus. We illustrate this in Fig. 3(b), where a detail of $V(r)$ for blocks $p=10$ and $p=11$ is shown, for $D=0.0048$, which verifies $D_{C}^{(10)} \leq D \leq D_{C}^{(11)}$.

Moreover, a signature of the block structured dynamics still persist for large noise levels, as shown by the curve for $V(r)$ at $D=0.100$ [bottom curve of Figs. 3(a) and 3(c)]. This comes from the above mentioned decrease of the centers of oscillation within each block and shows up as a straight line with slope $\Delta V / \Delta(\ln r) \approx-3.9 \times 10^{2}$ in the $V$ versus $\ln (r)$ plot.

The outputs of experiments are ISI sequences; therefore, other statistical quantities are easily accessible experimentally and deserve to be considered. We present three of them in Fig. 4 for two extreme cases of the noise strength, $D=0$ and $D=0.1$, and an intermediate value appropriate for each case. The mean $\langle T\rangle$ appears in Fig. 4(a) for the intermediate value $D=0.0040$; the skewness,

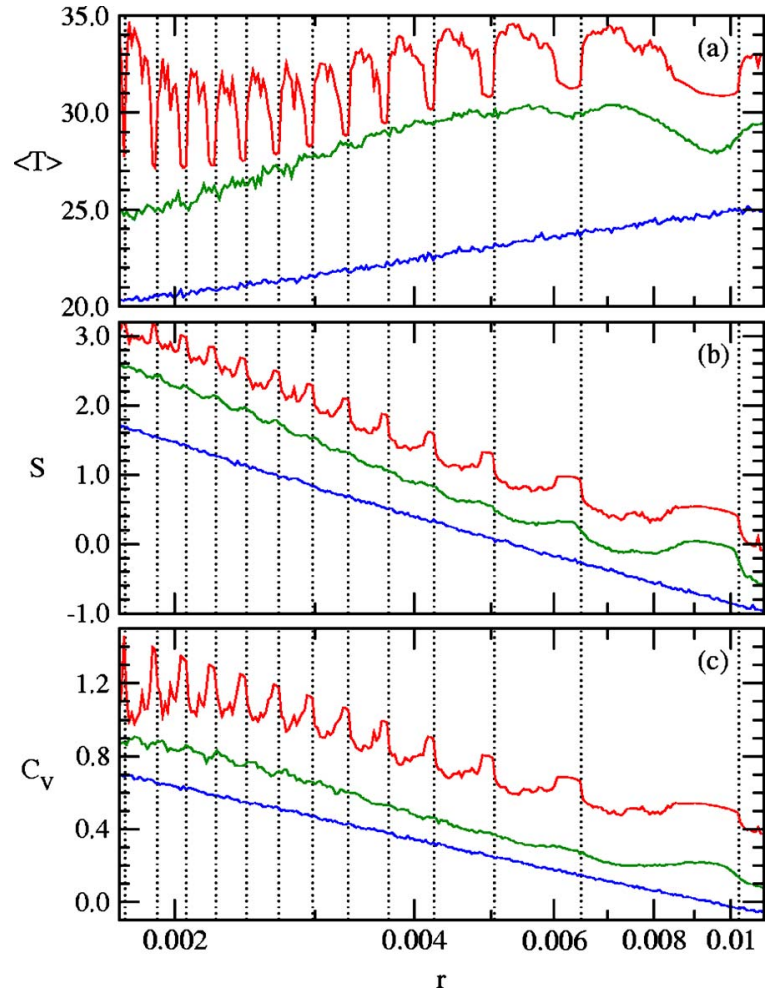

FIG. 4. (Color online) Statistical quantities characterizing the ISI distribution as functions of $r$ for representative values of the noise strength. (a) The mean for $D=0$ (top), $D=0.0040$ (middle), and $D=0.100$ (bottom). The last two curves are shifted down the same amount, 3.5, to make the figure clear. (b) The skewness for $D=0$ (top), $D=0.0015$ (middle), and $D=0.100$ (bottom). The last two curves are shifted down 0.5 and 1.5 , respectively. (c) The coefficient of variation for $D=0$ (top), $D=0.0035$ (middle), and $D=0.100$ (bottom). The last two curves are shifted down 0.3 and 0.6. Quantities plotted are dimensionless, $\langle T\rangle$ is measured in units of time $\tau, S$ and $C_{V}$ are pure numbers (no units), and $r$ is measured in units of reciprocal time $\tau^{-1}$.

$$
S=\frac{1}{N} \sum_{j=1}^{N}\left[\left(\frac{T_{j}-\langle T\rangle}{\sqrt{V}}\right)\right]^{3},
$$

in Fig. 4(b) for $D=0.0015$; and the coefficient of variation, $C_{V}=\sqrt{V} /\langle T\rangle$, in Fig. 4(c) for $D=0.0035$. The qualitative behaviors observed in the three cases are essentially the same seen above for the variance, with an oscillation inside each block and a certain shift of the centers of the oscillations. This shift is approximately linear for $S$ and $C_{V}$ plotted versus $\ln (r)$, but has a more complex structure for $\langle T\rangle$. Two other quantitative differences are relevant: the overall slope of the several curves and the ability of the observables to preserve signatures of the block structured dynamics against the effect of noise. To ease comparison, the slopes have been estimated from the curves for $D=0.1$. In the normalized form given, the ratio $R=\Delta Q /\left[Q_{15} \Delta(\ln r)\right]$, with $Q \in\left\{\langle T\rangle, S, C_{V}, V\right\}$ being the quantity considered, and $Q_{15}$ as the value of $Q$ at the limit between blocks $p=15$ and $p=14$. This ratio is $R \approx 0.10$ for $\langle T\rangle, R \approx 0.32$ for $C_{V}, R \approx 0.41$ for $V$, and $R \approx 0.47$ for $S$. Otherwise, for levels of noise allowing us 
to use a stair-like dependence as in Fig. 3(b), we have found that the skewness, for which $0.15 \leqq D_{C}^{(p)} \lesssim 0.25$ is the weakest, while the mean $\left(0.40 \leqq D_{C}^{(p)} \lessgtr 0.60\right)$ and the coefficient of variation $\left(0.35 \leq D_{C}^{(p)} \leq 0.50\right)$ rate similar to the variance in this issue.

\section{NEURONAL CODING}

We will now show that the behavior of observables like $V(r)$ may contribute to our understanding of how neurons encode information. Let us start with a more formal presentation of the relevant results shown in Fig. 3. For a level of noise $D_{m}$ smaller than $D_{C}^{(p)}$, but close to it, we can idealize the dependence of $V$ on $r$, by means of a surrogate variance, $v(r)$, and its uncertainty function, $u(r)$. To define these functions, we consider an interval which begins at $r_{I}^{(p)}$ and ends at $r_{F}^{(p)}$, with $r_{I}^{(p)}$ being the smallest value of $r<r_{M}^{(p)}$ which verifies $v(r) \leqslant V_{M}^{(p)}$, and $r_{F}^{(p)}$ as the largest value of $r>r_{m}^{(p)}$ which verifies $V(r) \geqslant V_{m}^{(p)}$. Once defined, these intervals for a block, $v(r)$ and $u(r)$, are given by $v(r)=\left[r_{F}^{(p)}-r_{I}^{(p)}\right]^{-1} \int_{r_{I}^{(p)}}^{r_{F}^{(p)}} V(r) d r$ $\equiv v^{(p)} \quad$ and $\quad u(r)=V_{M}^{(p)}-V_{m}^{(p)} \equiv \delta v^{(p)}$, both for $r \in \Delta^{(p)}$ $\equiv\left[r_{I}^{(p)}, r_{F}^{(p)}\right]$. These functions are defined only in the set of points enclosed in $\Delta^{(p)}$. In practice, this is most of the range of variation of $r$ because $u(r)$ and $v(r)$ are to be used for $D$ in the neighborhood of a $D_{C}^{(p)}$ where $V(r)$ is approximately a stairs shaped function, so that $r_{I}^{(p-1)}-r_{F}^{(p)}$ is small compared to the length of $\Delta^{(p)}$. Moreover, we note that, for each block, $v(r)$ and $u(r)$ provide an appropriate coarse description of $V(r)$ for a range of values of $D$, up to certain value $D_{M}$, greater then $D_{C}^{(p)}$, but close to it. So, these functions are constructed to work as a coarse description of $V(r)$ in a narrow but finite range of values of noise around the set of values of $D_{C}^{(p)}$, at least for a subset of a few consecutive blocks. For example, in Fig. 3(b), rectangles of base $\Delta^{(p)}$ and height $\delta v^{(p)}$, around $v^{(p)}$, are plotted for $p=10$ and $p=11$.

Therefore, at least for a subset of consecutive blocks, the function $v(r)$ that takes a constant value $v^{(p)}$, inside $\Delta^{(p)}$, with a tolerance $\delta v^{(p)}$ equal to the value of $u(r)$ in $\Delta^{(p)}$ is a wellconstructed coarse description of $V(r)$. Provided that intervals $\delta v^{(p)}$ and $\delta v^{(p+1)}$, around $v^{(p)}$ and $v^{(p+1)}$, are separated by well-defined gaps, a coding mechanism can be defined. A common way to construct messages is by defining a set of code units ("letters") and then to make meaning units as strings of code units ("words"). The simplest set of code units is the binary code made up of two bits, which allows us to construct messages as sequences of $0 \mathrm{~s}$ and $1 \mathrm{~s}$. Therefore, a feasible hypothesis is that the neuronal code is made of words that convey meaning, which are composed of strings of letters. If this is to work, we need a mechanism that has two basic properties. One is accuracy: It must allow the generation of letters that have to be unambiguously distinguishable from one another. The other is reproducibility: It has to be possible to repeat precisely each letter as many times as needed.

The above block structures allow us to design a coding mechanism which has these fundamental properties by as- signing a different letter to each of the blocks available, so that any neuron whose $r$ is inside the interval $\Delta^{(p)}$ is sending the reproducible letter $v^{(p)}$. This is named by the value of $V(r) \approx v^{(p)}$ within $\delta v^{(p)}$, which is definitely different than the letter sent by other neuron working at $r$ in the interval $\Delta^{\left(p^{\prime}\right)} \neq \Delta^{(p)}$, which is characterized by $V(r) \approx v^{\left(p^{\prime}\right)} \neq v^{(p)}$, because $\delta v^{\left(p^{\prime}\right)}$ and $\delta v^{(p)}$ are disjointed intervals. Then accuracy is granted and precise words can be encoded by means of sequences of letters which can be unambiguously detected because of the stairs shape of $V(r)$. We give an example of this in Fig. 3(b), where $V(r)$ is displayed together with the intervals $\Delta^{(p)}$ and the tolerances $\delta v^{(p)}$ that define boxes in the $r$ - $V$ plane, which are the regions of neuronal working associated to the letters $v^{(10)}$ and $v^{(11)}$. Messages in binary code can be constructed working only within these two regions.

These ideas, with some changes, can also be applied to large levels of noise as illustrated in Fig. 3(c), where $V(r)$ in the region around the blocks $p=10$ and $p=11$ is plotted for $D=0.100$. Because signatures of the block structured dynamics still persist in the form of a monotonous decay with a large slope, it is possible to define boxes in the $r$-V plane, similar to those considered above, which will allow neuronal coding in a similar way. In this case, however, there is more freedom to choose the values of $\Delta, v$, and $\delta v$, which define each letter, because they do not have to be associated to blocks. We show in Fig. 3(c) an arbitrary choice of two boxes for which neuronal coding reasoning similar to that presented above can be done.

We note that, as illustrated by the results in Fig. 4, not all statistical quantities will rate equally for such a neural coding. A relevant feature is the overall slope, which has to be large to distinguish efficiently different letters, no matter if a stairs profile based code [Fig. 3(b)] or a slope based code [Fig. 3(c)] is used. The mean rates poorly in this issue, because of both the nonlinear shift of the centers of oscillation for low noise and the small slope for large noise. The other three quantities show a better behavior with the skewness getting the best result. Regarding to the resistance to noise needed for a coding based on a stair profile, the skewness, which can only be used for $D \leq 0.0020$, rates below the other three quantities, which allow this form of neural coding up to $D \approx 0.0050$.

Based on the above ideas, we speculate on possible neural coding systems that can generate time-varying stimulus that carry information. Varieties of neural systems exist, depending on the animal considered and on the task to be performed. Therefore, we do not expect a single mechanism of information processing that works for all cases, but a variety of them that could be designed using block structured dynamics. To illustrate this, we give two simple examples of input-output systems able to encode information, each based in one of the interpretations for the variation of the parameter $r$ given in Sec. II. For simplicity, we assume that the input is a certain stimulus $s$, which can take two values $s_{\alpha}$ and $s_{\beta}$. The output has to be a train of spikes which transmits the letter $v^{(p)}$ for $s_{\alpha}$ and $v^{(p+1)}$ for $s_{\beta}$.

In our first example [Fig. 5(a)], we assume that the value of $r$ of a neuron can be modified by means of the change of the concentration $c$ of some channel activating chemical. 

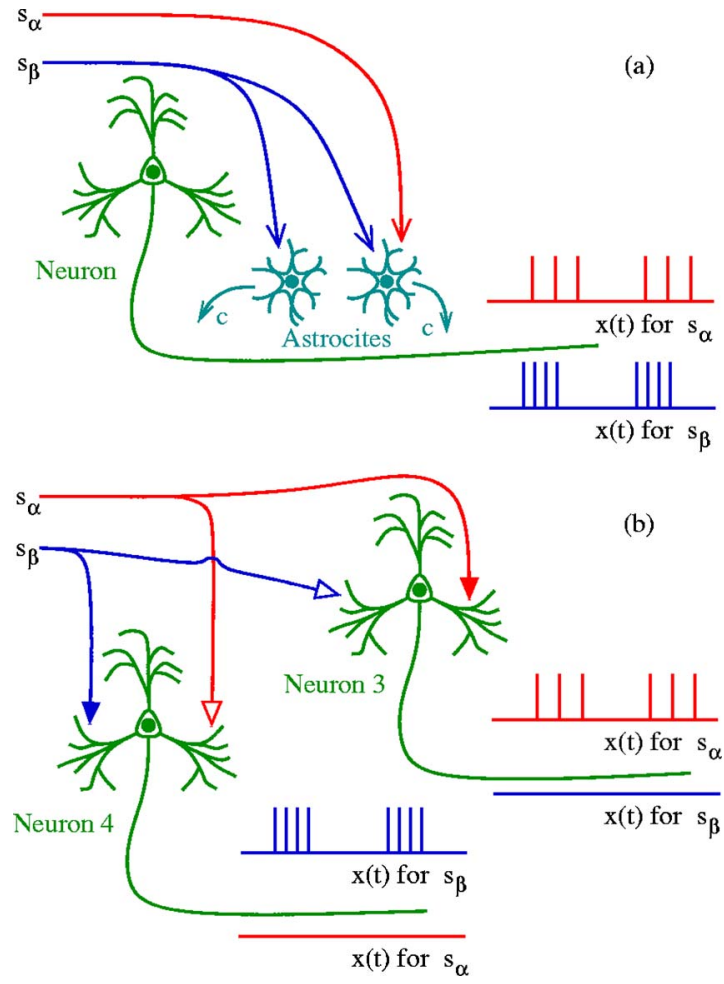

FIG. 5. (Color online) (a) The output of a neuron is modified by means of the change of the block where its dynamics occurs. The input from a stimulus which may take two values, $s_{\alpha}$ or $s_{\beta}$, activates the physiology of glial cells (astrocytes) to change the environmental concentration of some chemical able to modify the sensitivity of some ion gates in the neuron axon. (b) The activity of two different neurons that are working in different blocks is switched on and off by means of the stimulation of excitory (filled arrows) and inhibitory (open arrows) synapses. In the two cases, (a) and (b), the input $s_{\alpha}$ creates trains of spikes grouped in bursts of three; meanwhile, the input $s_{\beta}$ results in bursts of four spikes.

That means that for a series of values of $p$, there are values of the concentration $c_{p}$ with uncertainties $\Delta c_{p}$, such that when $c=c_{p} \pm \Delta c_{p}$, the neuron dynamics occurs in block $p$. Then, if $c$ is sensitive to the stimulus $s$, it is possible to change its value between $c_{\alpha} \approx c_{p} \pm \Delta c_{p}$ and $c_{\beta} \approx c_{p+1} \pm \Delta c_{p+1}$; then, $r$ changes between values within the intervals $\Delta^{(p)}$ and $\Delta^{(p+1)}$. This would determine the output of the neuron, which will be a train of spikes carrying the appropriate letter, $v^{(p)}$ or $v^{(p+1)}$, for the particular value of the stimulus, $s_{\alpha}$ or $s_{\beta}$. We guess the following biological mechanism for the realization of this transduction, which leans on the glial cells [25] that provide structural support, nourishment, and chemical environment regulation to the neurons. The stimulus $s_{\alpha}$ would act on some glial cells to modify the value of $c$ to $c_{\alpha}$; likewise, $s_{\beta}$ would change $c$ to $c_{\beta}$. Depending on the value of $s$, the appropriate output will result as a train of spikes along the neuron axon with dynamics in block $p$ or in block $p+1$. This biological mechanism for information transductions, which goes beyond direct synaptic stimulation, might seem unusual. This may be because, since their discovery, the glia was considered as a mere provider of support for the neurons. Forty years ago, some pioneering work [26,27] emphasized the role of glial cells in the genesis of field potentials.
Recently, based in these and other works, it has emerged the conviction that the neuroglia plays a significant role in neuronal information processing [28]. Because of this, we have introduced this possibility in our analyses.

In our second example [Fig. 5(b)], we understand the parameter $r$ as the value of a property of a single neuron that differentiates it from other neurons. For neural coding, this parameter tells us which letter, $v^{(p)}$, it is able to generate. The binary stimulus $s$ can be encoded by two different neurons, working in blocks $p$ and $p+1$, by activating or deactivating the appropriate neuron. Because the output of this system is the sum of the signals of the two neurons, in each case a constant membrane potential will add with a train of spikes giving a resulting signal with the appropriate statistics. A possible mechanism able to perform this task relies in the existence of excitory and inhibitory channel synapses [25]. That means that the input of a neuron on another can contribute positively or negatively to the generation of an action potential; then, the following arrangement that includes the two types of synapses would perform the desired switching: the stimulus $s_{\alpha}$ is transmitted by a nerve fiber that bifurcates providing an excitory input to a neuron working in block $p$ and an inhibitory input to the neuron working in block $p+1$. Similarly, the stimulus $s_{\beta}$ bifurcates to excite the neuron in block $p+1$ and inhibit the neuron in block $p$.

With the exception of primitive organisms, the activity of a nervous system is not performed by single neurons but by functional units made of a number of neurons. One of these units, whose job is to create certain messages, has to be made of at least a bunch of neurons that must have their values of $r$ distributed around the $r_{p}$ which is in the middle of one of the above intervals $\Delta^{(p)}$ with a dispersion much smaller than $\Delta^{(p)} / 2$. Nonchannel synaptic connections [25] would then be established only between neurons whose value of $r$ is within the same $\Delta^{(p)}$. If these connections are properly set, synchronization of the dynamics [29] of the neurons in the bunch can occur [13-17], and this will output a well-defined signal, characterized by a $v^{(p)}$ with a tolerance smaller than $\delta v^{(p)}$. Then, the functional unit would be organized into bunches of neurons, each being a differentiated element specialized in the generation of a letter $v^{(p)}$. A time-varying stimulus, $s_{\alpha}$, $s_{\beta}, \ldots$, can be encoded by these units by means of the above mechanisms: (i) changing the chemical environment to modify the value of $r$ in a single bunch, or (ii) having several bunches, each with its own $r$, and activating and deactivating them as needed. So, these functional units would generate accurate messages made of words composed of strings of letters: $v^{(a)} v^{(\beta)} \ldots$, which would be sequences of trains of spikes, each with the appropriate value of $v^{(p)}$. These timevarying signals would allow decoding by measuring a convenient quantity characterizing the ISI distribution. We note that for all this to work, the noise level must be stable; which is not a strong requirement if one has in mind the selfregulatory abilities of living beings.

\section{OTHER MODELS}

We note that block structured dynamics is not a particular property of the Hindmarsh-Rose model [8]. Other models of 
excitable cells display structures that are similar to those presented in Fig. 1. These include the model of Chay for a nerve cell [30], the modified Hodgkin-Huxley model of thermally sensitive neurons [31], and the Sherman model for a pancreatic $\beta$ cell [32].

We will illustrate this by means of some results for the Chay model [30], which is based on a Hodgkin-Huxley-type [9] formalism. In the formulation of this model, it is assumed that the membrane potential is determined by the combined dynamics of inward $\mathrm{Na}^{+}-\mathrm{Ca}^{2+}$ ion channels, outward $\mathrm{K}^{+}$ion channels (that can be of two types: voltage or chemical activated), and the average effect of other ionic channels, called leakage channels. The model has three dynamical variables: the membrane potential $V$, the probability of opening the $\mathrm{K}^{+}$voltage sensitive channels $n$, and the concentration of intracellular $\mathrm{Ca}^{2+}$ ions $C$. The dynamics equations are then

$$
\begin{gathered}
d V / d t=g_{I}^{*} m_{\infty}^{3} h_{\infty}\left(V_{I}-V\right)+g_{K, V}^{*} n^{4}\left(V_{K}-V\right) \\
+g_{K, C}^{*} \frac{C}{1+C}\left(V_{K}-V\right)+g_{L}^{*}\left(V_{L}-V\right), \\
d n / d t=\left(n_{\infty}-n\right) / \tau_{n}, \\
d C / d t=\rho\left[m_{\infty}^{3} h_{\infty}\left(V_{C}-V\right)-k_{C} C\right] .
\end{gathered}
$$

In these equations, $m_{\infty}, h_{\infty}$, and $n_{\infty}$ are steady state values of probabilities of activation or inactivation of voltage sensitive ion channels given by $x_{\infty}=a_{x} /\left(a_{x}+b_{x}\right)$, with $x \in\{m, h . n\}$, and

$$
\begin{gathered}
a_{m}=\frac{25+V}{10}\{1-\exp [-(25+V) / 10]\}^{-1}, \\
b_{m}=4 \exp [-(50+V) / 18], \\
a_{h}=(7 / 100) \exp [-(50+V) / 20], \\
b_{h}=\{1+\exp [-(20+V) / 10]\}^{-1}, \\
a_{n}=\frac{20+V}{100}\{1-\exp [-(20+V) / 10]\}^{-1}, \\
b_{n}=(1 / 8) \exp [-(30+V) / 80] .
\end{gathered}
$$

The relaxation time for $n$ is $\tau_{n}=\left[230\left(a_{n}+b_{n}\right)\right]^{-1}$. The parameters of the system are the reversal potentials $\left(V_{I}=100 \mathrm{mV}\right.$, $V_{K}=-75 \mathrm{mV}, V_{L}=-40 \mathrm{mV}$ ), and the maximal conductances divided by the membrane potential $\left(g_{I}^{*}=1800 \mathrm{~s}^{-1}, g_{K, V}^{*}\right.$ $\left.=1700 \mathrm{~s}^{-1}, g_{L}^{*}=7 \mathrm{~s}^{-1}\right)$ for the mixed $\mathrm{Na}^{+}-\mathrm{Ca}^{2+}$, the $\mathrm{K}^{+}$, and the leakage voltage sensitive ion channels, respectively, plus $k_{C}=3.3 / 18 \mathrm{mV}, \rho=0.27 \mathrm{mV}^{-1} \mathrm{~s}^{-1}$ and $V_{C}=100 \mathrm{mV}$. The control parameter of the model is the conductance for the $\mathrm{Ca}^{2+}$ sensitive $\mathrm{K}^{+}$ion channel, $g_{K, C}^{*}$, which we change in the range from $10.5 \mathrm{~s}^{-1}$ to $15.5 \mathrm{~s}^{-1}$. According to Chay [30], all these figures are in a range in which they can be taken as realistic values which allow qualitative reproduction of experimental results.
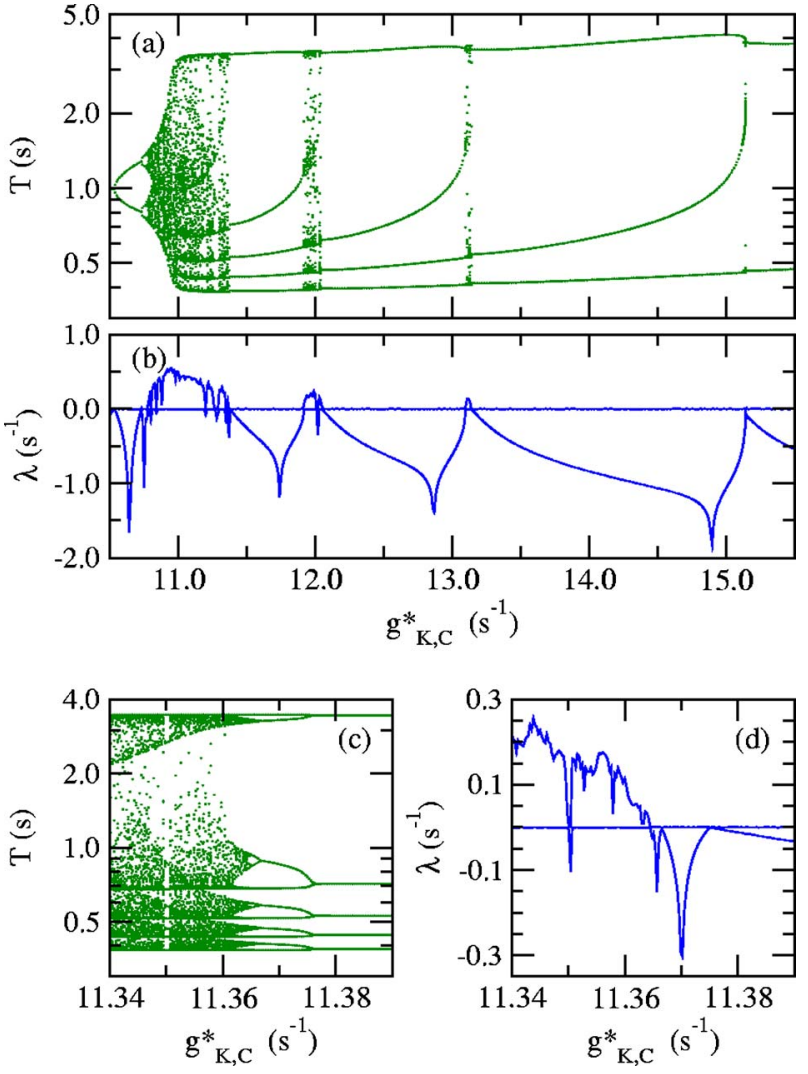

FIG. 6. (Color online) (a), (c) The Bifurcation diagram and (b), (d) the two largest Lyapunov exponents for the Chay model.

Our determination of the bifurcation diagram and the two largest Lyapunov exponents, presented in Fig. 6, show that after a continuous interior crisis that occurs at $g_{K, C}^{*} \approx 10.95 \mathrm{~s}^{-1}$, three blocks of dynamics, with periodicities 5,4 , and 3, develop as $g_{K, C}^{*}$ increases. This suggests that block structured dynamics may be common in systems of excitable cells, especially when parameters that describe the efficiency of ion channels are considered.

\section{CONCLUSIONS}

In summary, we have shown that there are block bifurcation structures in excitable systems, which are robust against noise and make it possible to encode messages in the random trains of spikes that run along a neuron axon. These structures allow the classification of the dynamics available to the system in classes, labeled by its periodicity. They may be common in models for the action potential that include control parameters describing the permeability of the membrane to certain ions. In a noisy environment, it is possible to find statistical observables that, despite the tendency of the noise to blur all structure, retain some features of the underlying block bifurcation structure. We have shown two potential ways to allow neuronal coding from these physical behaviors: one is based on a stairs profile for medium and low noise and the other on monotonous variations of appropriate observables for large noise. 
These results appear interesting for both physicists and biophysicists. Among other issues, the search for the conditions of existence of block structured dynamics, and the elucidation of the particular properties that these structures could induce in the physics of driven or coupled nonlinear oscillators, are of potential interest in nonlinear physics. Otherwise, the detailed biophysical mechanisms behind block structured dynamics, the extent of its presence in excitable cells, as well as all issues related to its application in neural coding, such as the choice of optimal observables or the elucidation of neuronal coding systems, are of interest in biophysics.

\section{ACKNOWLEDGMENT}

This research was supported by DGI (Grant No. BFM2003-05106).
[1] T. J. Sejnowski, Nature (London) 376, 21 (1995).

[2] E. E. Fetz, Science 278, 1901 (1997).

[3] D. Ferster and N. Spruston, Science 270, 756 (1995).

[4] A. V. Holden, Nature (London) 428, 382 (2004).

[5] M. J. Chacron, B. Lindner, and A. Longtin, Phys. Rev. Lett. 92, 080601 (2004).

[6] M. Abeles, Science 304, 523 (2004).

[7] Y. Ikegaya, G. Aaron, R. Cossart, D. Aronov, I. Lampl, D. Ferster, and R. Yuste, Science 304, 559 (2004).

[8] J. L. Hindmarsh and R. M. Rose, Proc. R. Soc. London, Ser. B 221, 87 (1984).

[9] A. L. Hodgkin and A. F. Huxley, J. Physiol. (London) 117, 500 (1952)

[10] A. I. Selverston, M. I. Rabinovich, H. D. I. Abarbanel, R. Elson, A. Szücs, R. D. Pinto, R. Huerta, and P. Varona, J. Physiol. (Paris) 94, 357 (2000).

[11] J. M. González-Miranda, Chaos 13, 845 (2003).

[12] S. Reinker, E. Puil, and R. M. Miura, Bull. Math. Biol. 65, 641 (2003).

[13] D. He, P. Shi, and L. Stone, Phys. Rev. E 67, 027201 (2003).

[14] R. Huerta, M. I. Rabinovich, H. D. I. Abarbanel, and M. Bazhenov, Phys. Rev. E 55, R2108 (1997).

[15] M. Dhamala, V. K. Jirsa, and M. Ding, Phys. Rev. Lett. 92, 028101 (2004).

[16] M. G. Rosenblum and A. S. Pikovsky, Phys. Rev. Lett. 92, 114102 (2004).

[17] I. Belykh, E. de Lange, and M. Hasler, Phys. Rev. Lett. 94, 188101 (2005).

[18] M. C. Eguia, M. I. Rabinovich, and H. D. I. Abarbanel, Phys.
Rev. E 62, 7111 (2000).

[19] P. E. Kloeden and E. Platen, Numerical Solution of Stochastic Differential Equations (Springer Verlag, New York, 1995).

[20] R. Fitzhugh, Biophys. J. 1, 445 (1961).

[21] H. G. Schuster, Deterministic Chaos (VCH Verlagsgesellschaft $\mathrm{mbH}$, Weinheim, 1988).

[22] R. Badii, E. Brun, M. Finardi, L. Flepp, R. Holzner, J. Parisi, C. Reyl, and J. Simonet, Rev. Mod. Phys. 66, 1389 (1994).

[23] W. H. Press, S. A. Teukolsky, W. T. Vetterling, and B. P. Flannery, Numerical Recipes in Fortran 77 (Cambridge University Press, Cambridge, UK, 1995).

[24] M. J. Chacron, A. Longtin, and L. Maler, J. Neurosci. 21, 5328 (2001).

[25] M. F. Bear, B. W. Connors, and M. A. Paradiso, Neuroscience: Exploring the Brain (Lippincott, Williams and Wilkins, Baltimore, MD, 2001).

[26] S. W. Kuffler, J. G. Nicholls, and R. K. Orkand, J. Neurophysiol. 29, 768 (1966).

[27] R. K. Orkand, J. G. Nicholls, and S. W. Kuffler, J. Neurophysiol. 29, 788 (1966).

[28] P. G. Haydon, Nat. Rev. Neurosci. 2, 185 (2001).

[29] J. M. González-Miranda, Synchronization and Control of Chaos (Imperial College Press, London, 2004).

[30] T. R. Chay, Physica D 16, 233 (1985).

[31] H. A. Braun, M. T. Huber, M. Dewald, K. Schäfer, and K. Voigt, Int. J. Bifurcation Chaos Appl. Sci. Eng. 8, 811 (1998).

[32] A. Sherman, J. Rinzel, and J. Keizer, Biophys. J. 54, 411 (1988). 